\title{
MORE ON AUTOMORPHISM GROUPS OF LAMINATED NEAR-RINGS
}

\author{
by D. K. BLEVINS, K. D. MAGILL, JR., P. R. MISRA, \\ J. C. PARNAMI and U. B. TEWARI
}

(Received 1st April 1986)

\section{Introduction}

We will assume throughout this paper that polynomials are nonconstant. Let $P$ be any complex polynomial and let $\mathscr{N}_{P}$ denote the near-ring of all continuous selfmaps of the complex plane where addition of functions is pointwise and multiplication is defined by $f g=f \circ P \circ g$ for all $f, g \in \mathcal{N}_{P}$. The near-ring $\mathscr{N}_{P}$ is referred to as a laminated near-ring and $P$ is referred to as the laminating element or laminator. In [1] the problem was posed of determining Aut $\mathscr{N}_{P}$ the automorphism group of $\mathscr{N}_{P}$. It was shown that exactly three infinite groups occur as automorphism groups of the laminated near-rings $\mathscr{N}_{P}$ and for each of the three groups those polynomials $P$ were characterized such that Aut $\mathcal{N}_{P}$ is isomorphic to that particular group. The infinite groups turn out to be $G L(2)$, the full linear group of all $2 \times 2$ nonsingular real matrices and two of its subgroups.

In [2], as the title of that paper indicates, finite automorphism groups of the nearrings $\mathscr{N}_{P}$ were investigated and the results obtained there, combined with the results obtained in [1], yielded a description of Aut $\mathcal{N}_{P}$ when $P$ has real coefficients and $\operatorname{Deg} P \leqq 4$. In this paper, we complete the solution of the problem. That is, the main result of this paper, together with a result from [1] allows us to completely describe Aut $\mathscr{N}_{P}$ with no restrictions whatsoever on the polynomial $P$. In Section 2 we recall some notation and state the main results. Proofs are given in Section 3.

\section{Statements of main results}

As we mentioned previously, $G L(2)$ denotes the full linear group of all real $2 \times 2$ nonsingular matrices. $G_{1}$ denotes the subgroup of $G L(2)$ consisting of all matrices of the form

$$
\left[\begin{array}{ll}
1, & a \\
0, & b
\end{array}\right] \text { where } b \neq 0
$$

and $G_{c}$ denotes the subgroup of $G L(2)$ consisting of all matrices of the form

$$
\left[\begin{array}{rr}
a, & -b \\
b, & a
\end{array}\right] \text { and }\left[\begin{array}{rr}
a, & b \\
b, & -a
\end{array}\right]
$$


where $a^{2}+b^{2} \neq 0$. Let $G R_{m}$ ( $m$ a positive integer) denote that subgroup of $G_{c}$ where $a=\cos (2 k \pi / m)$ and $b=\sin (2 k \pi / m), 1 \leqq k \leqq m$. Finally, we denote by $\mathbb{Z}_{m}$ the cyclic group of order $m$. The groups $G L(2), G_{1}$ and $G_{c}$ are all infinite while $G R_{m}$ is finite of order $2 m$. Of course, $G R_{1}$ is isomorphic to $\mathbb{Z}_{2}$ and $G R_{2}$ is isomorphic to $\mathbb{K}_{4}$ the Klein group of order four. These are the only instances in which $G R_{m}$ is abelian.

In all that follows, we let $P(z)=\sum_{j=0}^{n} a_{j} z^{n-j}$ where each $a_{j}$ is a complex number and $a_{0} \neq 0$. We now state two theorems which, together, completely describe Aut $\mathscr{N}_{P}$ for all complex polynomials $P$. The first result appears in [1] as Theorem 4.4. We restate it here (without proof) for the sake of completeness.

Theorem 2.1. Let $P$ be any complex polynomial. Then:

Aut $\mathscr{N}_{P}$ is isomorphic to $G L(2)$ if and only if $\operatorname{Deg} P=1$ or $\operatorname{Deg} P=2$ and $a_{1}=0$,

Aut $\mathscr{N}_{P}$ is isomorphic to $G_{1}$ if and only if $\operatorname{Deg} P=2$ and $a_{1} \neq 0$,

Aut $\mathcal{N}_{P}$ is isomorphic to $G_{\mathrm{c}}$ if and only if Deg $P \geqq 3$ and $a_{j}=0$ for $1 \leqq j \leqq n-1$.

It is evident from the previous result that it remains for us to consider the case where $\operatorname{Deg} P \geqq 3$ and $a_{j} \neq 0$ for some $j$ such that $1 \leqq j \leqq n-1$. We do this in the next theorem which is the main result of the paper. In the statement we require $a_{0}=1$. At first glance this may appear to be a restriction but it really is not for Lemma 3.2 of [1] assures us that Aut $\mathscr{N}_{P}$ and Aut $\mathscr{N}_{Q}$ are isomorphic where $Q(z)=\left(1 / a_{0}\right) P(z)$. In the statements of the following three results, we let $A=\left\{j: 1 \leqq j \leqq n-1\right.$ and $\left.a_{j} \neq 0\right\}$.

Theorem 2.2. Let $\operatorname{Deg} P \geqq 3$ and $a_{0}=1$. Suppose $A \neq \varnothing$ and let $m=\operatorname{gcd} A$. Then there exist integers $b_{j}$ such that $m=\sum_{j \in A} j b_{j}$ and we define

$$
c=\prod_{j \in A}\left(a_{j} / \bar{a}_{j}\right)^{b_{j}} .
$$

If there exists an mth root $\sigma$ of $c$ such that

$$
a_{j}=\bar{a}_{j} \sigma^{j} \text { for each } j \in A,
$$

then Aut $\mathcal{N}_{P}$ is isomorphic to $G R_{m}$. If no mth root of c satisfies (2.2.2), then Aut $\mathscr{N}_{P}$ is isomorphic to $\mathbb{Z}_{m}$ the cyclic group of order $m$.

Corollary 2.3. Let $\operatorname{Deg} P \geqq 3$ and let $a_{0}=1$. Suppose $A \neq \varnothing$ and $a_{j}$ is real for each $j \in A$. Then Aut $\mathscr{N}_{\mathrm{P}}$ is isomorphic to $G R_{m}$ where $m=\operatorname{gcd} A$.

Corollary 2.4. Let $\operatorname{Deg} P \geqq 3$, let $a_{0}=1$, suppose $A \neq \varnothing$ and suppose $a_{j}$ is a pure imaginary number for each $j \in A$. Suppose further that the least element $m \in A$ divides every other element in $A$. Then:

Aut $\mathscr{N}_{P}$ is isomorphic to $G R_{m}$ if $j / m$ is odd for each $j \in A$. 
and

$$
\text { Aut } \mathscr{N}_{P} \text { is isomorphic to } \mathbb{Z}_{m} \text { if } j / m \text { is even for at least one } j \in A \text {. }
$$

\section{Supporting lemmas and proofs}

Lemma 3.1. Let $P(z)$ be any complex polynomial and let any real number $r>0$ be given. Then there exists an $R>0$ such that if $\left|z_{0}\right|>R$, then $z_{0}$ is the only zero of $P(z)-P\left(z_{0}\right)$ in the interior of the curve $C=\left\{z:\left|z-z_{0}\right|=r\right\}$.

Proof. Let $Q(z)=P(z)-P\left(z_{0}\right)$. We then have

$$
Q(z)=\sum_{j=0}^{n} \frac{Q^{(j)}\left(z_{0}\right)}{j !}\left(z-z_{0}\right)^{j}=\sum_{j=1}^{n} \frac{P^{(j)}\left(z_{0}\right)}{j !}\left(z-z_{0}\right)^{j} .
$$

According to Lemma 3.4 of [1], we can choose $R_{1}$ large enough so that if $\left|z_{0}\right|>R_{1}$, then $Q(z)$ has no multiple roots. Thus, $Q^{\prime}\left(z_{0}\right)=P^{\prime}\left(z_{0}\right) \neq 0$ for $\left|z_{0}\right|>R_{1}$ and from this fact and (3.1.1) we get

$$
Q(z)=P^{\prime}\left(z_{0}\right)\left(z-z_{0}\right)[1+R(z)]
$$

where

$$
R(z)=\sum_{j=2}^{n} \frac{P^{(j)}\left(z_{0}\right)}{j ! P^{\prime}\left(z_{0}\right)}\left(z-z_{0}\right)^{j-1} .
$$

Now let $\varepsilon$ be any number such that $0<\varepsilon<1$ and choose $R_{2} \geqq R_{1}$ so that $|R(z)|<\varepsilon$ when $\left|z_{0}\right|>R_{2}$ and $z$ is any point on the curve $C$. From this and (3.1.2) we get

$$
|Q(z)| \geqq r\left|P^{\prime}\left(z_{0}\right)\right|(1-\varepsilon)
$$

for any $z$ on $C$. Since $P^{\prime}\left(z_{0}\right) \neq 0$, this means, among other things, that $Q(z)$ does not vanish on $C$.

In a similar manner,

$$
Q^{\prime}(z)=P^{\prime}\left(z_{0}\right)[1+T(z)]
$$

where

$$
T(z)=\sum_{j=1}^{n-1} \frac{P^{(j+1)}\left(z_{0}\right)}{j ! P^{\prime}\left(z_{0}\right)}\left(z-z_{0}\right)^{j}
$$

Now choose $R \geqq R_{2}$ so that if $\left|z_{0}\right|>R$ and $z$ is any point on $C$, then $|T(z)|<\varepsilon$. It follows from (3.1.5) that

$$
\left|Q^{\prime}(z)\right| \leqq\left|P^{\prime}\left(z_{0}\right)\right|(1+\varepsilon)
$$


From (3.1.4) and (3.1.7) we then get

$$
\begin{aligned}
\left|\frac{1}{2 \pi i} \int_{c} \frac{Q^{\prime}(z)}{Q(z)} d z\right| & \leqq \frac{1}{2 \pi} \int_{0}^{2 \pi} \frac{\left|Q^{\prime}(z)\right|}{|Q(z)|} r d \theta \\
& \leqq \frac{1}{2 \pi} \int_{0}^{2 \pi} \frac{\left|P^{\prime}\left(z_{0}\right)\right|(1+\varepsilon)}{r\left|P^{\prime}\left(z_{0}\right)\right|(1-\varepsilon)} r d \theta=\frac{1+\varepsilon}{1-\varepsilon}
\end{aligned}
$$

The number $\varepsilon$ can be chosen small enough so that $(1+\varepsilon) /(1-\varepsilon)<2$ and it then follows from the Principle of the Argument that $z_{0}$ is the only zero of $Q(z)=P(z)-P\left(z_{0}\right)$ within the curve $C$ when $\left|z_{0}\right|>R$.

We next recall a result of J. L. Walsh [3, p. 21] which we will need in the proof of a subsequent lemma.

Theorem 3.2 (Walsh). Let $P(z)=\left(z-\alpha_{1}\right)\left(z-\alpha_{2}\right) \ldots\left(z-\alpha_{n}\right)$ and let $\alpha_{0}=\left(\alpha_{1}+\alpha_{2}+\cdots\right.$ $\left.+\alpha_{n}\right) / n$. For each $\varepsilon>0$ there exists an $M_{\varepsilon}$ such that if $|A|>M_{\varepsilon}$ then every zero $z_{0}$ of the polynomial $P(z)-A$ satisfies an inequality

$$
\left|z_{0}-\left(\alpha_{0}+A^{1 / n}\right)\right|<\varepsilon
$$

where $A^{1 / n}$ is a suitably chosen $n$th root of $A$.

Any polynomial $P$ decomposes the complex plane $\mathscr{C}$ into mutually disjoint subsets. Specifically, we define

$$
\Pi(P)=\left\{P^{-1}(w): w \in \mathscr{C}\right\} .
$$

Next, we regard $\mathscr{C}$ as a two-dimensional vector space over the reals and we denote by $L A(P)$ the group of all linear automorphisms $t$ of $\mathscr{C}$ which satisfy the condition $t[A] \in \Pi(P)$ for each $A \in \Pi(P)$. Corollary (2.3) of [1] tells us that Aut $\mathscr{N}_{P}$ is isomorphic to $L A(P)$ so our efforts in this section will be directed toward determining $L A(P)$ for each complex polynomial $P$. There is another result we need to recall from [1]. It was stated there as Lemma 3.1.

Lemma 3.3. A linear automorphism $t$ of $\mathscr{C}$ belongs to $L A(P)$ if and only if for all $z_{1}, z_{2} \in \mathscr{C}$, the following two statements are equivalent:

$$
\begin{gathered}
P\left(z_{1}\right)=P\left(z_{2}\right) . \\
P\left(t\left(z_{1}\right)\right)=P\left(t\left(z_{2}\right)\right) .
\end{gathered}
$$

And now we are in a position to prove

Lemma 3.4. Let $P(z)=z^{n}+a_{1} z^{n-1}+a_{2} 2^{n-2}+\cdots+a_{n}$, let $t \in L A(P)$ and let $\theta$ be $a$ primitive $n$th root of unity. Then the sets

$$
\left\{t(1), t(\theta), t\left(\theta^{2}\right), \ldots, t\left(\theta^{n-1}\right)\right\}
$$


and

$$
\left\{t(1), \theta t(1), \theta^{2} t(1), \ldots, \theta^{n-1} t(1)\right\}
$$

coincide.

Proof. According to Lemma 3.1 we can choose $R_{0}>0$ so that if $\left|z_{0}\right|>R_{0}$ then $z_{0}$ is the only zero of $P(z)-P\left(z_{0}\right)$ within the curve $\left|z-z_{0}\right|=1$. Next let $\varepsilon$ be given subject to the conditions $0<\varepsilon<\frac{1}{2}$. According to Walsh's Theorem 3.2, there exists an $M_{\varepsilon}$ such that if $|A|>M_{\varepsilon}$ then every zero $z_{0}$ of $P(z)-A$ satisfies (3.2.1). Next, let $M=$ $\sup _{|z| \leqq R_{0}}|P(z)|$ and then choose $R_{\varepsilon}$ so that whenever $|z|>R_{\varepsilon}$, the following three conditions are satisfied.

$$
\begin{gathered}
\left|P\left(z_{0}\right)\right|>M+M_{\varepsilon} \\
\left|P\left(t\left(z_{0}\right)\right)\right|>M_{\varepsilon} \\
P(z)-P\left(z_{0}\right) \text { has } n \text { distinct zeros. }
\end{gathered}
$$

It is evident that the first two conditions can be satisfied and it follows from Lemma 3.4 of [1] that the third condition can be satisfied as well.

Now let $l>R_{\varepsilon}$, let $|P(l)|^{1 / n}$ be the positive $n$th root of $|P(l)|$ and let $z_{1}$ be any zero of $P(z)-P(l)$. It follows from (3.4.3) and Walsh's Theorem that

$$
\left|z_{1}-\left(\alpha_{0}+|P(l)|^{1 / n} \theta^{j}\right)\right|<\varepsilon
$$

where $1 \leqq j \leqq n$. Let $z_{2}$ be a zero of $P(z)-P(l)$ distinct from $z_{1}$. As in the case for $z_{1}$ we have

$$
\left|z_{2}-\left(\alpha_{0}+|P(l)|^{1 / n} \theta^{i}\right)\right|<\varepsilon
$$

where $1 \leqq i \leqq n$ and we claim that $i \neq j$. Suppose, to the contrary, that $i=j$. It follows from (3.4.6) and (3.4.7) that

$$
\left|z_{1}-z_{2}\right|<2 \varepsilon<1
$$

Thus, $P(z)-P\left(z_{1}\right)=P(z)-P(l)$ has at least two zeros within $\left|z-z_{1}\right|=1$. But from (3.4.3) we see that $\left|P\left(z_{1}\right)\right|=|P(l)|>M$ which implies $\left|z_{1}\right|>R_{0}$. This, in turn, implies that $P(z)-P\left(z_{1}\right)$ has only one zero within $\left|z-z_{1}\right|=1$ and we have reached a contradiction. Consequently, $i \neq j$ as we asserted. According to (3.4.5), $P(z)-P(l)$ has $n$ distinct zeros and it follows from all this that for each integer $j$ such that $1 \leqq j \leqq n$, there exists a zero $z_{l j}$ of $P(z)-P(l)$ such that

$$
\left|z_{l j}-\left(\alpha_{0}+|P(l)|^{1 / n} \theta^{j}\right)\right|<\varepsilon
$$


Thus, we have

$$
\lim _{l \rightarrow \infty}\left|z_{l j}-\left(\alpha_{0}+\mid P(l)^{1 / n} \theta^{j}\right)\right|=0
$$

for each $j$. This implies

$$
\lim _{l \rightarrow \infty}\left|\frac{z_{l j}}{l}-\frac{|P(l)|^{1 / n}}{l} \theta^{j}\right|=0
$$

But

$$
\lim _{l \rightarrow \infty} \frac{|P(l)|}{l^{n}}=1 \quad \text { so that } \quad \lim _{l \rightarrow \infty} \frac{|P(l)|^{1 / n}}{l}=1
$$

This, together with (3.4.11) implies

$$
\lim _{l \rightarrow \infty} \frac{z_{l j}}{l}=\theta^{j} \quad \text { for } \quad 1 \leqq j \leqq n .
$$

Since $t$ is continuous, we also have

$$
\lim _{l \rightarrow \infty} \frac{t\left(z_{l j}\right)}{l}=t\left(\theta^{j}\right) \text { for } \quad 1 \leqq j \leqq n
$$

It follows from Lemma 3.3 that $\left\{t\left(z_{i j}\right)\right\}_{j=1}^{n}$ is the collection of zeros for the polynomial $P(z)-P(t(l))$. Choose any $t\left(z_{i j}\right)$. Since $l>R_{\varepsilon},\left|P\left(t\left(z_{i j}\right)\right)\right|=|P(t(l))|>M_{\varepsilon}$ and it follows from Walsh's Theorem that

$$
\left|t\left(z_{l j}\right)-\left(\alpha_{0}+P(t(l))^{1 / n}\right)\right|<\varepsilon
$$

where $P(t(l))^{1 / n}$ is a suitable $n$th root of $P(t(l))$. This implies

$$
\lim _{l \rightarrow \infty}\left|\frac{t\left(z_{l j}\right)}{\operatorname{lt}(1)}-\frac{P(t(l))^{1 / n}}{l t(1)}\right|=0
$$

Thus, (3.4.13) and (3.4.15) together imply that

$$
\lim _{l \rightarrow \infty} \frac{P(t(l))^{1 / n}}{l t(1)}=\frac{t\left(\theta^{j}\right)}{t(1)}
$$

But we have

$$
\lim _{t \rightarrow \infty}\left[\frac{P(t(l))^{1 / n}}{l t(1)}\right]^{n}=\lim _{l \rightarrow \infty} \frac{P(t(l))}{(t(l))^{n}}=1
$$


so that $\left[t\left(\theta^{j}\right) / t(1)\right]^{n}=1$. That is, $t\left(\theta^{j}\right) / t(1)=\theta^{i}$ for some $i$ such that $1 \leqq i \leqq n$. Thus, $t\left(\theta^{j}\right)=\theta^{i} t(1) \in\left\{t(1), \theta t(1), \ldots, \theta^{n-1} t(1)\right\}$ for $1 \leqq j \leqq n$. Consequently, the sets (3.4.1) and (3.4.2) coincide as we asserted.

Lemma 3.5. Let $P(z)=z^{n}+a_{1} z^{n-1}+a_{2} z^{n-2}+\cdots+a_{n}$ with $\operatorname{Deg} P \geqq 3$ and let $t \in L A(P)$. Then there exists a nonzero complex number $v$ such that either $t(z)=v z$ for all $z \in \mathscr{C}$ or $t(z)=v \bar{z}$ for all $z \in \mathscr{C}$.

Proof. We first consider the case where $\operatorname{Deg} P=4$. Then, by Lemma (3.4) we have

$$
t(i) \in\{t(1), i t(1),-t(1),-i t(1)\}
$$

If $t(i)=t(1)$, then $0=t(i)-t(1)=t(i-1)$ which is a contradiction. Thus, $t(i) \neq t(1)$ and for similar reasons, $t(i) \neq-t(1)$. Consequently, either $t(i)=i t(1)$ or $t(i)=-i t(1)$. In the former case,

$$
\begin{aligned}
t(x+y i) & =t(x)+t(y i)=x t(1)+y t(i) \\
& =x t(1)+y i t(1)=t(1)(x+y i)
\end{aligned}
$$

and one shows in the same manner that $t(x+y i)=t(1) \overline{(x+y i)}$ in the latter case.

Now suppose $\operatorname{Deg} P \neq 4$ and let $\theta=x+y i$ be a primitive $n$th root of unity where $n=$ $\operatorname{Deg} P$. Then $x \neq 0 \neq y$ (since $\operatorname{Deg} P \geqq 3$ and $\operatorname{Deg} P \neq 4$ ) and $x^{2} \neq 1$. The vectors $1, \theta$ and $\bar{\theta}$ all have absolute value 1 and by Lemma $3.4, t(1), t(\theta)$ and $t(\bar{\theta})$ all have absolute value $|t(1)|$. It now follows from Lemma 4.1 of [1] that there exists a nonzero complex number $v$ such that either $t(z)=v z$ for each $z \in \mathscr{C}$ or $t(z)=v \bar{z}$ for each $z \in \mathscr{C}$.

Notation. Let $v$ be a nonzero complex number. In all that follows $t_{v}$ is the linear automorphism of $\mathscr{C}$ which is defined by $t_{v}(z)=v z$ and $\hat{t}_{v}$ is defined by $\hat{t}_{v}(z)=v \bar{z}$. As before, we let $A=\left\{j: 1 \leqq j \leqq n-1\right.$ and $\left.a_{j} \neq 0\right\}$ and we assume $A \neq \varnothing$. Furthermore, we assume without further mention that $\operatorname{Deg} P \geqq 3$ and $a_{0}=1$.

Lemma 3.6. $t_{v} \in L A(P)$ if and only if $v^{j}=1$ for each $j \in A$.

Proof. Suppose $t_{v} \in L A(P)$ and choose $z_{1}$ so that $P^{-1}\left(P\left(z_{1}\right)\right)$ consists of $n$ distinct points $\left\{z_{j}\right\}_{j=1}^{n}$. Then we have

$$
P(z)-P\left(z_{1}\right)=\left(z-z_{1}\right)\left(z-z_{2}\right) \ldots\left(z-z_{n}\right) .
$$

Now $\left\{z_{j}\right\}_{j=1}^{n} \in \Pi(P)$ so $\left\{v z_{j}\right\}_{j=1}^{n} \in \Pi(P)$ since $t_{v} \in L A(P)$. It follows that $P^{-1}\left(P\left(v z_{1}\right)\right)=$ $\left\{v z_{j}\right\}_{j=1}^{n}$ and this implies

$$
P(z)-P\left(v z_{1}\right)=\left(z-v z_{1}\right)\left(z-v z_{2}\right) \ldots\left(z-v z_{n}\right)
$$

From (3.6.1) and (3.6.2), we get

$$
P(v z)-P\left(v z_{1}\right)=v^{n}\left[P(z)-P\left(z_{1}\right)\right]
$$


Choose $j \in A$. The coefficient of $z^{n-j}$ in $P(v z)-P\left(v z_{1}\right)$ is $v^{n-j} a_{j}$ and the coefficient of $z^{n-j}$ in $v^{n}\left[P(z)-P\left(z_{1}\right)\right]$ is $v^{n} a_{j}$. It follows from (3.6.3) that $v^{n-j} a_{j}=v^{n} a_{j}$ for each $j \in A$ and this implies $v^{j}=1$ for each $j \in A$.

Conversely, suppose that $v^{j}=1$ for each $j \in A$. Then we have

$$
\begin{aligned}
P(v z) & =a_{n}+v^{n} z^{n}+\sum_{j \in A} a_{j} v^{n-j} z^{n-j} \\
& =a_{n}+v^{n} z^{n}+v^{n} \sum_{j \in A} a_{j} z^{n-j} \\
& =a_{n}+v^{n}\left[P(z)-a_{n}\right] .
\end{aligned}
$$

It readily follows from (3.6.4) that for any $z_{1}, z_{2} \in \mathscr{C}$, we have $P\left(z_{1}\right)=P\left(z_{2}\right)$ if and only if $P\left(v z_{1}\right)=P\left(v z_{2}\right)$. Thus, $t_{v} \in L A(P)$ by Lemma 3.3.

Lemma 3.7. $\hat{t}_{v} \in L A(P)$ if and only if $a_{j} / \bar{a}_{j}=v^{j}$ for each $j \in A$.

Proof. Suppose $\hat{t}_{v} \in L A(P)$. Again choose $z_{1}$ so that $P^{-1}\left(P\left(z_{1}\right)\right)$ consists of $n$ distinct elements $\left\{z_{i}\right\}_{i=1}^{n}$. As before, we have

$$
P(z)-P\left(z_{1}\right)=\left(z-z_{1}\right)\left(z-z_{2}\right) \ldots\left(z-z_{n}\right)
$$

and this time $\left\{v \bar{v}_{i}\right\}_{i=1}^{n} \in \Pi(P)$ which implies

$$
P(z)-P\left(v \bar{z}_{1}\right)=\left(z-v \bar{z}_{1}\right)\left(z-v \bar{z}_{2}\right) \ldots\left(z-v \bar{z}_{n}\right) .
$$

From (3.7.1) and (3.7.2), we get

$$
P(v \vec{z})-P\left(v \bar{z}_{1}\right)=v^{n} \overline{\left[P(z)-P\left(z_{1}\right)\right]} .
$$

For each $j \in A$, the coefficient of $\bar{z}^{n-j}$ in $P(v \bar{z})-P\left(v \bar{z}_{1}\right)$ is $a_{j} v^{n-j}$ while the coefficient of $\bar{z}^{n-j}$ in $v^{n}\left[\overline{\left.P(z)-P\left(z_{1}\right)\right]}\right.$ is $v^{n} \bar{a}_{j}$. Thus, $a_{j} v^{n-j}=v^{n} \bar{a}_{j}$ by (3.7.3) and it follows that $a_{j} / \bar{a}_{j}=v^{j}$ for each $j \in A$.

Suppose, conversely, that $\left(a_{j} / \bar{a}_{j}\right)=v^{j}$ for each $j \in A$. We then have

$$
\begin{aligned}
P(v \bar{z}) & =a_{n}+v^{n} \bar{z}^{n}+\sum_{j \in A} a_{j} v^{n-j} \bar{z}^{n-j} \\
& =a_{n}+v^{n} \bar{z}^{n}+v^{n} \sum_{j \in \mathrm{A}} \bar{a}_{j} \bar{z}^{n-j} \\
& =a_{n}+v^{n}\left[P(\bar{z})-a_{n}\right] .
\end{aligned}
$$

It readily follows from (3.7.4) that for any $z_{1}, z_{2} \in \mathscr{C}$ we have $P\left(z_{1}\right)=P\left(z_{2}\right)$ if and only if $P\left(v \bar{z}_{1}\right)=P\left(v \bar{z}_{2}\right)$ and we appeal to Lemma 3.3 once again to conclude that $\hat{t}_{v} \in L A(P)$.

Notation. We let $B(P)=\left\{v \in \mathscr{C}: t_{v} \in L A(P)\right\}$ and $\hat{B}(P)=\left\{v \in \mathscr{C}: \hat{t}_{v} \in L A(P)\right\}$.

Lemma 3.8. Let $m=\operatorname{gcd} A$ and let $\theta$ be a primitive mth root of unity. Then $B(P)=$ $\left\{\theta^{i}\right\}_{i=1}^{m}$. 
Proof. Suppose $v \in B(P)$. Then $t_{v} \in L A(P)$ and $v^{j}=1$ for each $j \in A$ by Lemma 3.6. Furthermore, there exist integers $\left\{b_{j}\right\}_{j \in A}$ so that $m=\sum_{j \in A} j b_{j}$ and we have

$$
v^{m}=\prod_{j \in A}\left(v^{j}\right)^{b_{j}}=1
$$

That is, $v$ is an $m$ th root of unity. Since $\theta$ is a primitive $m$ th root, we have $\theta^{k}=v$ for some $k$ where $1 \leqq k \leqq m$. On the other hand, it is immediate from Lemma 3.6 that for each such $k$, we have $\theta^{k} \in B(P)$.

Lemma 3.9. Let $m=\operatorname{gcd} A$. Then there exist integers $b_{j}$ such that $m=\sum_{j \in A} j b_{j}$ and we define

$$
c=\prod_{j \in A}\left(a_{j} / \bar{a}_{j}\right)^{b_{j}} .
$$

If there exists an $m$ th root $\sigma$ of $c$ such that

$$
a_{j}=\bar{a}_{j} \sigma^{j} \text { for all } j \in A \text {, }
$$

then

$$
\widehat{B}(P)=\left\{\sigma \theta^{i}\right\}_{i=1}^{m}
$$

where $\theta$ is a primitive $m$ th root of unity. If no $m$ th root of $c$ satisfies (3.9.2), then

$$
\hat{B}(P)=\varnothing \text {. }
$$

Proof. Suppose $\sigma$ satisfies (3.9.2). Then $\theta^{j}=1$ for each $j \in A$ and we get $\left(\sigma \theta^{i}\right)=a_{j} / \bar{a}_{j}$ for each $j \in A$ which, by Lemma 3.7, means $\sigma \theta^{i} \in \hat{B}(P)$. On the other hand, suppose $v \in \hat{B}(P)$. Then $a_{j} / \bar{a}_{j}=v^{j}$ for each $j \in A$. We use (3.9.1) and get

$$
v^{m}=\prod_{j \in A}\left(v^{J}\right)^{b_{j}}=\prod_{j \in A}\left(a_{j} / \bar{a}_{j}\right)^{b_{j}}=c .
$$

That is, $v$ is an $m$ th root of $c$ and it follows that $v=\sigma \theta^{i}$ for some $i$ such that $1 \leqq i \leqq m$.

To prove the last assertion of the lemma, deny it and suppose $v \in \hat{B}(P)$. Then $a_{j} / \bar{a}_{j}=v^{j}$ for each $j \in A$ by Lemma 3.7. From (3.9.1) we get $v^{m}=c$ just as before. But this is a contradiction since we now have an $m$ th root of $c$ which satisfies (3.9.2). Therefore, we conclude that $\hat{B}(P)=\varnothing$ when no $m$ th roots of $c$ satisfy (3.9.2).

Our next result shows that the two sets $B(P)$ and $\widehat{B}(P)$ do not intersect except under very special circumstances.

Corollary 3.10. The following statements are equivalent:

$$
\begin{gathered}
B(P) \cap \hat{B}(P) \neq \varnothing \\
B(P)=\hat{B}(P) .
\end{gathered}
$$


Proof. We show (3.10.1) implies (3.10.3). Suppose $v \in B(P) \cap \hat{B}(P)$. Then $a_{j} / \bar{a}_{j}=1$ for each $j \in A$ by Lemmas 3.6 and 3.7. Thus, $a_{j}$ is real for each $j \in A$ and, of course, $a_{0}=1$. It is only $a_{n}$ that may possibly not be a real number.

Next, we show that (3.10.3) implies (3.10.2). We appeal to Lemma 3.9. In that lemma, $c=1$ and we take $\sigma=1$. Then (3.9.2) is satisfied and $\hat{B}(P)=\left\{\theta^{i}\right\}_{i=1}^{m}$ by (3.9.3). It now follows from Lemma 3.8 that $B(P)=\hat{B}(P)$. It is evident (since $B(P) \neq \varnothing$ ) that $(3.10 .2$ ) implies (3.10.1) and the proof is complete.

Notation. Let $\theta$ be a primitive $m$ th root of unity. We let $G_{m}(\theta)=\left\{t_{v}, \hat{t}_{v}: v=\theta^{i}\right.$, $1 \leqq i \leqq m\} . G_{m}(\theta)$ is, of course, a finite subgroup of the group of linear automorphisms of $\mathscr{C}$.

Lemma 3.11. Let $m=\operatorname{gcd} A$. Then there exist integers $b_{j}$ such that $m=\sum_{j \in A} j b_{j}$ and we define

$$
c=\prod_{j \in A}\left(a_{j} / \bar{a}_{j}\right)^{b_{j}}
$$

Suppose there exists an $m$ th root $\sigma$ of $c$ such that

$$
a_{j}=\bar{a}_{j} \sigma^{j} \text { for all } j \in A \text {. }
$$

Then $L A(P)$ is isomorphic to $G_{m}(\theta)$.

Proof. Since $a_{j} / \bar{a}_{j}=\sigma^{j}$, we have $\bar{a}_{j} / a_{j}=\bar{\sigma}^{j}$ which implies $(\sigma \bar{\sigma})^{j}=1$. Now $\sigma \bar{\sigma}$ is a positive real number so we must have $\sigma \bar{\sigma}=1$. According to Lemmas 3.5, 3.8 and 3.9,

$$
L A(P)=\left\{t_{v}: v=\theta^{i}, 1 \leqq i \leqq m\right\} \cup\left\{\hat{t}_{w}: W=\sigma \theta^{i}, 1 \leqq i \leqq m\right\}
$$

Since $\sigma \bar{\sigma}=1$, one easily verifies that the mapping $\phi$ from $L A(P)$ to $G_{m}(\theta)$ defined by $\phi\left(t_{v}\right)=t_{v}$ and $\phi\left(\hat{t}_{w}\right)=\hat{t}_{u}$ where $u=\theta^{i}$ whenever $w=\sigma \theta^{i}$, is an isomorphism.

It is now an easy matter to complete the proof of Theorem 2.2 and to derive its corollaries. Suppose first that there exists an $m$ th root of $c$ satisfying condition (2.2.2) of Theorem 2.2. According to Lemma 3.11, $L A(P)$ is isomorphic to $G_{m}(\theta)$ and one easily verifies that if $v=\theta^{k}, 1 \leqq k \leqq m$, the map which sends $t_{v}$ to

$$
\left[\begin{array}{rr}
\cos (2 k \pi / m), & -\sin (2 k \pi / m) \\
\sin (2 k \pi / m), & \cos (2 k \pi / m)
\end{array}\right] \text { and } \hat{t}_{v} \text { to }\left[\begin{array}{rr}
\cos (2 k \pi / m), & \sin (2 k \pi / m) \\
\sin (2 k \pi / m), & -\cos (2 k \pi / m)
\end{array}\right]
$$

is an isomorphism from $G_{m}(\theta)$ onto $G R_{m}$. It now follows from Corollary 2.3 of [1] that in this particular case, Aut $\mathscr{N}_{P}$ is isomorphic to $G R_{m}$.

Now consider the remaining case where no $m$ th root of $c$ satisfies (2.2.2). It follows from Lemmas $3.5,3.8$ and 3.9 that $L A(P)=\left\{t_{v}: v=\theta^{i}, 1 \leqq i \leqq m\right\}$ which is cyclic of order $m$. Consequently, in this case Aut $\mathscr{N}_{P}$ is isomorphic to $\mathbb{Z}_{m}$. 
Corollary 2.3 follows easily from Theorem 2.2 . One has only to observe that if $a_{j}$ is real for every $j \in A$, then $c=1$ and one can then choose $\sigma=1$ and (2.2.2) is satisfied. As for Corollary 2.4 , we take $b_{m}=1$ and $b_{j}=0$ for all $j \in A-\{m\}$. Then $c$, as defined by (2.2.1), is -1 . Choose any $m$ th root $\sigma$ of -1 . Condition (2.2.2) will be satisfied if and only if $j / m$ is odd for each $j \in A$. Consequently, it follows from Theorem 2.2 that Aut $\mathcal{N}_{P}$ is isomorphic to $G R_{m}$ if $j / m$ is odd for each $j \in A$ and Aut $\mathscr{N}_{P}$ is isomorphic to $\mathbb{Z}_{m}$ if $j / m$ is even for at least one $j \in A$.

\section{REFERENCES}

1. K. D. Magill, P. R. Misra and U. B. Tewari, Automorphism groups of laminated near-rings determined by complex polynomials, Proc. Edinburgh Math. Soc. 26 (1983), 73-84.

2. K. D. Magill, P. R. Misra and U. B. TewarI, Finite automorphism groups of laminated near-rings, Proc. Edinburgh Math. Soc. 26 (1983), 297-306.

3. J. L. WALSH, The Location of Critical Points of Analytic and Harmonic Functions (Colloquium Pub. Vol. 34, Amer. Math. Soc., New York, 1950).

Epistemos, Inc.

Quaker Hill, Connecticut 06375

U.S.A.

College of Staten IsLand

Staten Island, New York 10301

U.S.A.

IIT KANPUR

KanPUR-208016, U.P.

INDIA
SUNY at Buffalo

Buffalo, New York 14214-3093

U.S.A.

Punjab University

Chandigarh 160014

INDIA 